\title{
IncRNA-CCHE1 is involved in migration and invasion but not in proliferation of pancreatic adenocarcinoma cells possibly by interacting with ROCK1
}

\author{
XIANG JIN ${ }^{1}$, LIPING YE ${ }^{1}$, MINHUA LIN ${ }^{1}$, BINBIN GU $^{1}$, JUN WANG ${ }^{1}$, YEQUN HE $^{1}$ and WEILIN LI ${ }^{2}$ \\ Departments of ${ }^{1}$ Gastroenterology and ${ }^{2}$ Urology, Taizhou Hospital of Zhejiang Province, \\ Wenzhou Medical University, Taizhou, Zhejiang 317000, P.R. China
}

Received August 30, 2018; Accepted February 12, 2019

DOI: $10.3892 / 01.2019 .10416$

\begin{abstract}
Cervical carcinoma high-expressed long non-coding RNA (lncRNA) 1 (lncRNA-CCHE1) serves an oncogenic role in cervical and liver cancer. The present study aimed to explore the role of CCHE1 in pancreatic adenocarcinoma. CCHE1 expression was detected by reverse transcription-quantitative polymerase chain reaction, and rho associated coiled-coil containing protein kinase 1 (ROCK1) levels were detected using an ELISA assay. Diagnostic analysis was performed by receiver operating characteristic curve analysis. The effects of CCHE1 on ROCK1 were analyzed by western blotting. Cell migration and invasion were analyzed by Transwell migration and invasion assays. The results of the present study demonstrated that, compared with healthy controls, CCHE1 and ROCK1 were upregulated in the serum of patients with metastatic pancreatic adenocarcinoma. CCHE1 overexpression distinguished patients with metastatic pancreatic adenocarcinoma from patients with non-metastatic pancreatic adenocarcinoma and healthy controls. A significant positive correlation between serum levels of CCHE1 and ROCK1 was identified in patients with metastatic pancreatic adenocarcinoma. Furthermore, CCHE1 overexpression led to upregulated ROCK1 in human pancreatic adenocarcinoma cell lines, whereas no significant effects of ROCK1 overexpression upon CCHE1 expression were identified. CCHE1 overexpression promoted the migration and invasion of human pancreatic adenocarcinoma cell lines, but no significant effects on cell proliferation were identified. ROCK1 small interfering RNA-induced silencing partially reversed the enhancing effects of CCHE1 overexpression on cancer
\end{abstract}

Correspondence to: Dr Weilin Li, Department of Urology, Taizhou Hospital of Zhejiang Province, Wenzhou Medical University, 150 Ximen Street, Linhai, Taizhou, Zhejiang 317000, P.R. China E-mail:nqpdnf5@163.com

Key words: pancreatic adenocarcinoma, metastasis, cervical carcinoma high-expressed long non-coding RNA 1, rho associated coiled-coil containing protein kinase 1 cell migration and invasion. Therefore, lncRNA-CCHE1 may be involved in migration and invasion but not in proliferation of pancreatic adenocarcinoma cells, possibly by interacting with ROCK1.

\section{Introduction}

Pancreatic cancer is characterized by its aggressive nature and high mortality rate (1). In different regions of the world, the mortality rate of this disease almost reaches its incidence rate $(2,3)$. Compared with patients with non-metastatic pancreatic cancer, the survival conditions of patients with metastatic pancreatic cancer are poor (4). No significant improvements in the survival rates of patients with metastatic pancreatic cancer have been made during the past several decades (4). It has been predicted that pancreatic cancer will become the second leading cause of cancer-associated mortality in the next decade in certain regions, including the United States (5). Despite the high mortality rate of patients with metastatic pancreatic cancer, the survival time can be markedly prolonged by appropriate treatment. Therefore, a proper treatment strategy design based on the detection of metastatic tumors is critical.

Rho associated coiled-coil containing protein kinase 1 (ROCK1) is a well-characterized transcription factor that serves a pivotal oncogenic role in cancer biology (6-8). Development of cancer in humans is usually accompanied by the activation of ROCK1 signaling (9), and inhibition of ROCK1 expression inhibits cancer development $(10,11)$. Cervical carcinoma high-expressed long non-coding RNA (lncRNA) 1 (lncRNA-CCHE1) serves an oncogenic role in cervical and liver cancer $(12,13)$. Preliminary microarray data revealed that IncRNA-CCHE1 and ROCK1 expression are upregulated and positively correlated in patients with metastatic pancreatic adenocarcinoma (data not shown, patients included in the microarray analysis were also involved in the patient group in this study). These findings indicated a potential interaction between them. The present study revealed that IncRNA-CCHE1 may be involved in the migration and invasion of cancer cells in pancreatic adenocarcinoma, which is the most common type of pancreatic cancer. 


\section{Materials and methods}

Cell lines and human samples. Capan-2 [American Type Culture Collection (ATCC) HTB-80 ${ }^{\mathrm{TM}}$ ] and HPAF-II (ATCC CRL-1997 ${ }^{\mathrm{TM}}$ ) human pancreatic adenocarcinoma cell lines were purchased from ATCC (Manassas, VA, USA). Cells were cultured in ATCC-formulated McCoy's 5a medium (cat. no. 30-2007; ATCC), supplemented with $10 \%$ fetal bovine serum (Sangon Biotech Co., Ltd., Shanghai, China) at $37^{\circ} \mathrm{C}$ in a $5 \% \mathrm{CO}_{2}$. incubator.

Human serum samples were derived from the blood extracts of 63 patients with pancreatic adenocarcinoma and 44 healthy volunteers. The 63 patients were diagnosed for the first time and admitted to Taizhou Hospital of Zhejiang Province (Taizhou, China) between May 2016 and May 2018. Among these patients, tumor metastasis was observed in 30 cases, which were classified as the metastasis group. The remaining patients were classified as the non-metastasis group. There were 18 cases of American Joint Committee on Cancer stage I, 20 cases of stage II, 12 cases of stage III and 13 cases of stage IV cancer (14). The 44 healthy volunteers received physical examination at the Physical Examination Center of Taizhou Hospital of Zhejiang Province during the same time period. No significant differences in the distribution of age and sex, as well as smoking and drinking habits, among the three groups were identified (Table I). The present study was approved by the Ethics Committee of Taizhou Hospital of Zhejiang Province. All participants fully understood the experimental protocol and signed informed consent forms.

Reverse transcription-quantitative polymerase chain reaction $(R T-q P C R)$. Total RNA was extracted from serum and in vitro cultivated cells using RNAzol ${ }^{\circledR}$ reagent (Sigma-Aldrich; Merck KGaA, Darmstadt, Germany), and subjected to reverse transcription, which was performed using SuperScript III Reverse Transcriptase (Thermo Fisher Scientific, Inc., Waltham, MA, USA) under the following thermal conditions: $25^{\circ} \mathrm{C}$ for $5 \mathrm{~min}$, $55^{\circ} \mathrm{C}$ for $20 \mathrm{~min}$ and $75^{\circ} \mathrm{C}$ for $5 \mathrm{~min}$. Subsequently, cDNA samples and a SuperScript III Platinum One-Step RT-qPCR kit (Thermo Fisher Scientific, Inc.) were used to prepare all PCR reactions. PCR reactions were performed using the following thermocycling conditions: $95^{\circ} \mathrm{C}$ for $50 \mathrm{sec}$, followed by $95^{\circ} \mathrm{C}$ for $12 \mathrm{sec}$ and $58.5^{\circ} \mathrm{C}$ for $20 \mathrm{sec}$ for 40 cycles; no final extension. Sequences of primers used in the PCR system were: lncRNA-CCHE1 forward, 5'-AAGGTCCCAGGATACTCG C-3' and reverse, 5'-GTGTCGTGGACTGGCAAAAT-3'; and $\beta$-actin forward, 5'-GACCTCTATGCCAACACAGT-3' and reverse, 5'-AGTACTTGCGCTCAGGAGGA-3'. Quantification cycle $(\mathrm{Cq})$ values of IncRNA-CCHE1 were normalized to the endogenous control $\beta$-actin using the $2^{-\Delta \Delta \mathrm{Cq}}$ method (15).

ELISA. The ROCK1 ELISA kit used for the measurement of serum levels of ROCK1 was obtained from MyBioSource, Inc. (cat. no. MBS9318720; MyBioSource, Inc., San Diego, CA, USA). This kit has a detection sensitivity of $0.1 \mathrm{pg} / \mathrm{ml}$ and a detection range of $2.5-80 \mathrm{ng} / \mathrm{ml}$.

Vectors, small interfering RNAs (siRNAs) and cell transfection. pIRSE2 vectors expressing full-length
Table I. General information regarding the three groups.

\begin{tabular}{lccc}
\hline Variable & $\begin{array}{c}\text { Metastasis } \\
(\mathrm{n}=30)\end{array}$ & $\begin{array}{c}\text { Non-metastasis } \\
(\mathrm{n}=33)\end{array}$ & $\begin{array}{c}\text { Control } \\
(\mathrm{n}=44)\end{array}$ \\
\hline Age range, years & $34-66$ & $33-67$ & $33-68$ \\
Mean age, years & $50.2 \pm 5.6$ & $50.7 \pm 5.2$ & $50.5 \pm 5.8$ \\
Sex & & & \\
$\quad$ Male & 16 & 16 & 21 \\
Female & 14 & 17 & 23 \\
Habits & & & \\
Smoking & $14(46.7 \%)$ & $14(42.4 \%)$ & $20(45.5 \%)$ \\
Drinking & $16(53.3 \%)$ & $17(51.5 \%)$ & $22(50.0 \%)$ \\
\hline
\end{tabular}

lncRNA-CCHE1 and ROCK1 were synthesized by Shanghai GenePharma Co., Ltd. (Shanghai, China). ROCK1 siRNA (cat. no. AM16708) and Silencer ${ }^{\circledR}$ Negative Control No. 2 siRNA (cat. no. AM4613) were purchased form Thermo Fisher Scientific, Inc. According to the manufacturer's protocols, cell transfection was performed using Lipofectamine ${ }^{\circledR} 2000$ (cat. no. 11668-019, Invitrogen; Thermo Fisher Scientific, Inc.) with vectors and siRNAs at dosages of 15 and $50 \mathrm{nM}$, respectively. The cell density for transfection was $10^{5} \mathrm{cell} / \mathrm{s} / \mathrm{ml}$. In the present study, cells transfected with empty vectors or siRNA control were considered the negative control groups. The control groups included cells without transfection. Overexpression rates of lncRNA-CCHE1 and ROCK1 were $>160 \%$, and ROCK 1 knockdown rate $>55 \%$ in response to ROCK1 siRNA $12 \mathrm{~h}$ after transfection. Transfection efficiency was detected by RT-qPCR (data not shown). Cells were collected $12 \mathrm{~h}$ after transfection prior to subsequent experimentation.

Cell proliferation assay. Following transfection, cell proliferation rates of different groups of cells were measured by Cell Counting kit-8 (CCK-8; Sigma-Aldrich; Merck KGaA) assay. Briefly, cells were harvested to prepare single cell suspensions $\left(3 \times 10^{4}\right.$ cells $\left./ \mathrm{ml}\right)$. A 96-well plate was filled with single cell suspension $(0.1 \mathrm{ml} / \mathrm{well})$. Cells were cultured at $37^{\circ} \mathrm{C}$ in a $\mathrm{CO}_{2}$. incubator, followed by the addition of $10 \mu \mathrm{l} \mathrm{CCK}-8$ solution $24,48,72$ and $96 \mathrm{~h}$ later. Following incubation for an additional $4 \mathrm{~h}$, absorbance at $450 \mathrm{~nm}$ was measured using Fisherbrand ${ }^{\mathrm{TM}}$ accuSkan $^{\mathrm{TM}}$ GO UV/Vis Microplate Spectrophotometer (Thermo Fisher Scientific, Inc.).

In vitro cell migration and invasion assays. Following transfection, cell migration and invasion rates of different groups of cells were measured by Transwell migration and invasion assays. The materials used, including the Transwell inserts (96 wells; pore size, $8 \mu \mathrm{m}$; cat. no. CLS3374), were obtained from Sigma-Aldrich; Merck KGaA. Briefly, cells were harvested to prepare serum-free signal cell suspensions $\left(3 \times 10^{4} \mathrm{cell} / \mathrm{s} / \mathrm{ml}\right)$. A total of $100-\mu \mathrm{l}$ serum-free cell suspension in ATCC-formulated McCoy's 5a medium was added to the upper chamber and cell culture medium containing $20 \%$ fetal calf serum (Sigma-Aldrich; Merck KGaA) was added into the lower chamber. After $12 \mathrm{~h}$, membranes were stained with 


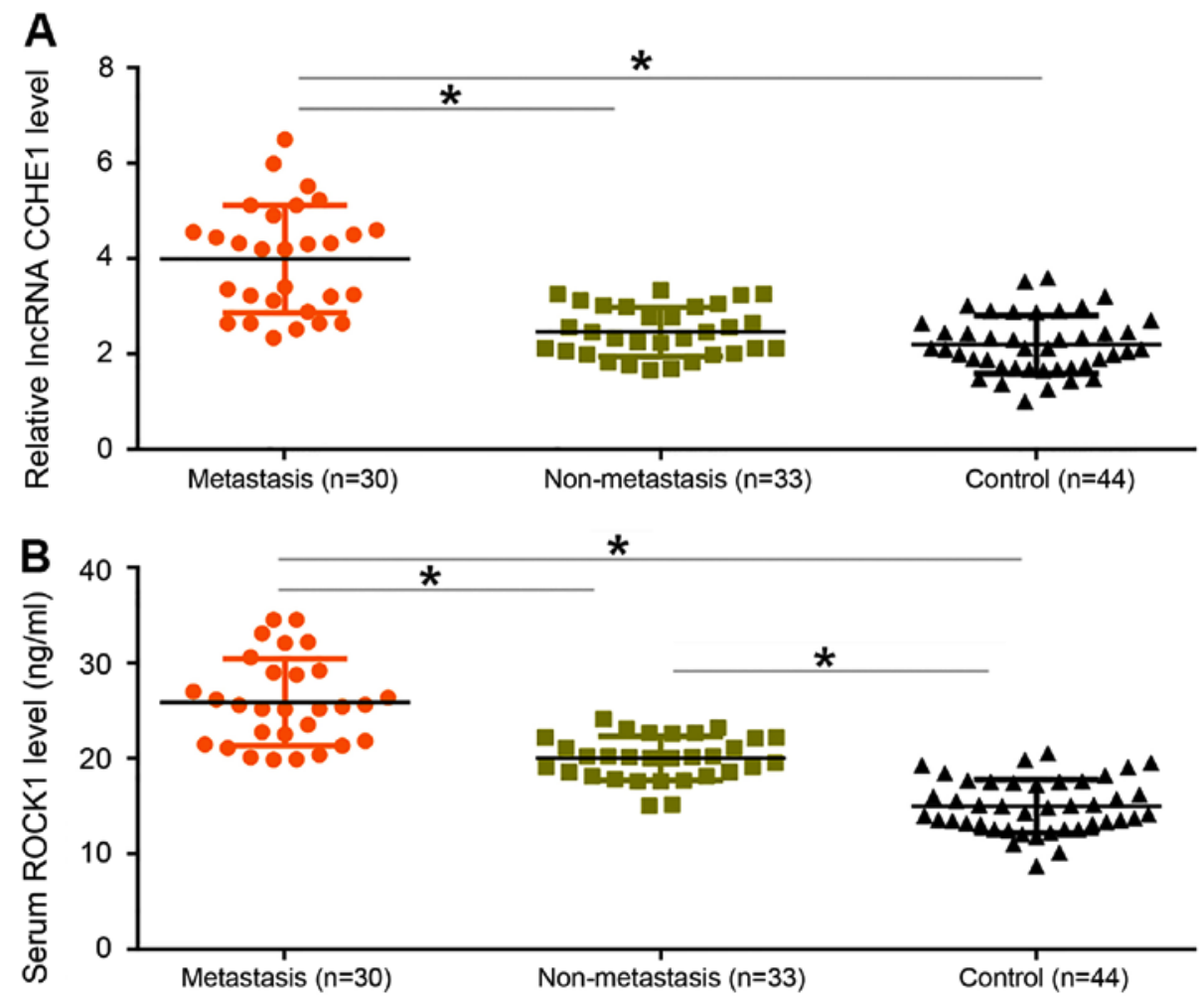

Figure 1. Upregulated serum lncRNA-CCHE1 and ROCK1 are observed in patients with metastatic pancreatic adenocarcinoma. Levels of (A) CCHE1 and (B) ROCK1 were significantly increased in patients with metastatic pancreatic adenocarcinoma compared with in patients with non-metastatic pancreatic adenocarcinoma and healthy controls. ${ }^{*} \mathrm{P}<0.05$. CCHE1, cervical carcinoma high-expressed lncRNA 1; lncRNA, long non-coding RNA; ROCK1, rho associated coiled-coil containing protein kinase 1 .

$0.5 \%$ crystal violet (Sigma-Aldrich; Merck KGaA) at $25^{\circ} \mathrm{C}$ for $15 \mathrm{~min}$. Migration and invasion assays were performed using the same protocol with the exception that the upper chamber was pre-coated with $50 \mu \mathrm{g}$ Matrigel in culture medium prior to the invasion assay. Migratory and invasive cells in the lower chamber were observed under a light microscope. The number of invasive and migratory cells in the control group was set to $100 \%$ and other groups were normalized to the control group.

Western blotting. Following total protein extraction using radioimmunoprecipitation assay buffer (Sangon Biotech Co., Ltd.) and concentration measurement using the bicinchoninic acid method, electrophoresis was performed using $12 \%$ SDS-PAGE with $30 \mu \mathrm{g}$ protein loaded per lane. Subsequently, transfer to polyvinylidene difluoride (PVDF) membranes was performed, followed by blocking in $5 \%$ skimmed milk at $25^{\circ} \mathrm{C}$ for $1.5 \mathrm{~h}$. PVDF membranes were incubated with ROCK1 (rabbit anti-human; 1:1,500 dilution; cat. no. ab97592; Abcam, Cambridge, UK) and GAPDH (rabbit anti-human; 1:1,200 dilution; cat. no. ab37168; Abcam) primary antibodies at $4^{\circ} \mathrm{C}$ for $8 \mathrm{~h}$, followed by incubation with immunoglobulin $\mathrm{G}$-horseradish peroxidase secondary antibody (goat anti-rabbit; 1:1,500 dilution; cat. no. MBS435036; MyBioSource, Inc.) at $25^{\circ} \mathrm{C}$ for $2 \mathrm{~h}$. Pierce Enhanced Chemiluminescence Western Blotting Substrate (Thermo Fisher Scientific, Inc.) was used to develop signals that were scanned by MYECL ${ }^{\mathrm{TM}}$ Imager (Thermo Fisher Scientific, Inc.). ImageJ v1.6 software
(National Institutes of Health, Bethesda, MD, USA) was used for data normalization.

Statistical analysis. GraphPad Prism v6 software (GraphPad Software, Inc., La Jolla, CA, USA) was used for statistical analysis in the present study. All experiments were performed in triplicate and data are expressed as the means \pm standard deviation. Data comparisons were performed by one-way analysis of variance followed by Tukey's post hoc testing. Pearson correlation coefficient was used for correlation analyses. The diagnostic value of lncRNA-CCHE1 for pancreatic adenocarcinoma was evaluated by receiver operating characteristic (ROC) curve analysis. $\mathrm{P}<0.05$ was considered to indicate a statistically significant difference.

\section{Results}

Serum lncRNA-CCHE1 and ROCK1 are upregulated in patients with metastatic pancreatic adenocarcinoma. Compared with in patients with non-metastatic pancreatic adenocarcinoma and healthy controls, the expression levels of CCHE1 (Fig. 1A) and ROCK1 (Fig. 1B) were significantly increased in patients with metastatic pancreatic adenocarcinoma $(\mathrm{P}<0.05)$. In addition, serum levels of ROCK1 were significantly higher in patients with non-metastatic pancreatic adenocarcinoma compared with in healthy controls.

Upregulation of IncRNA-CCHE1 distinguishes patients with metastatic pancreatic adenocarcinoma from patients with 

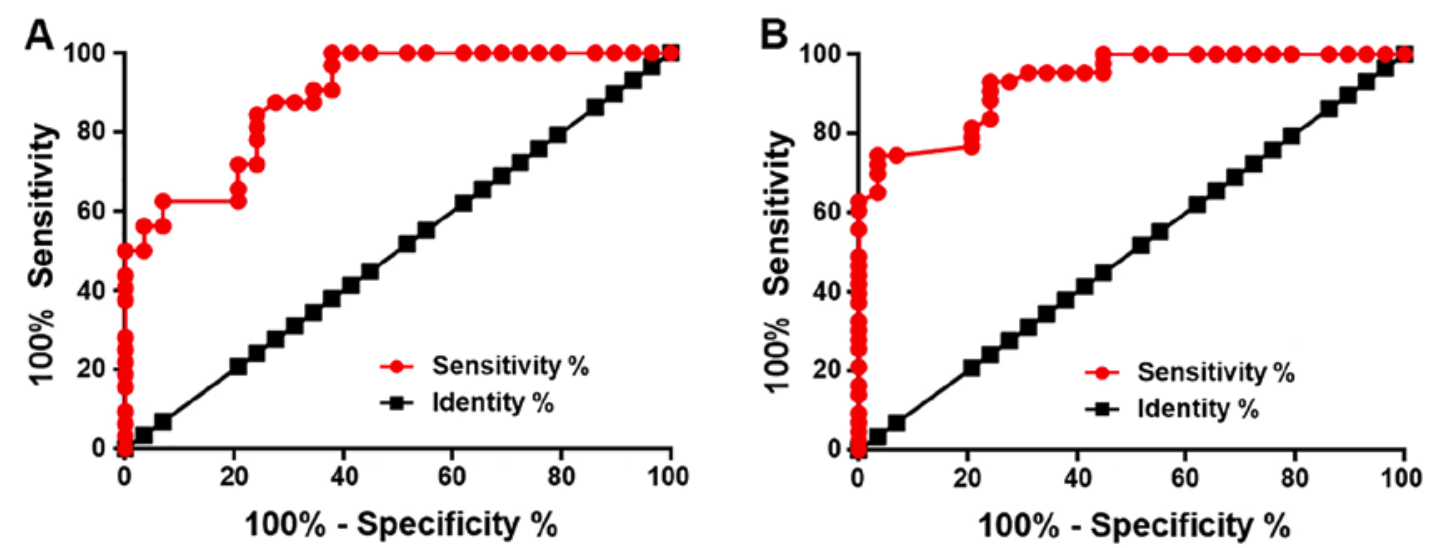

Figure 2. Upregulation of lncRNA-CCHE1 distinguishes patients with metastatic pancreatic adenocarcinoma from patients with non-metastatic pancreatic adenocarcinoma and healthy controls. Receiver operating characteristic curve analysis revealed that upregulation of lncRNA-CCHE1 distinguished patients with metastatic pancreatic adenocarcinoma from (A) patients with non-metastatic pancreatic adenocarcinoma and (B) healthy controls. CCHE1, cervical carcinoma high-expressed lncRNA 1; lncRNA, long non-coding RNA.
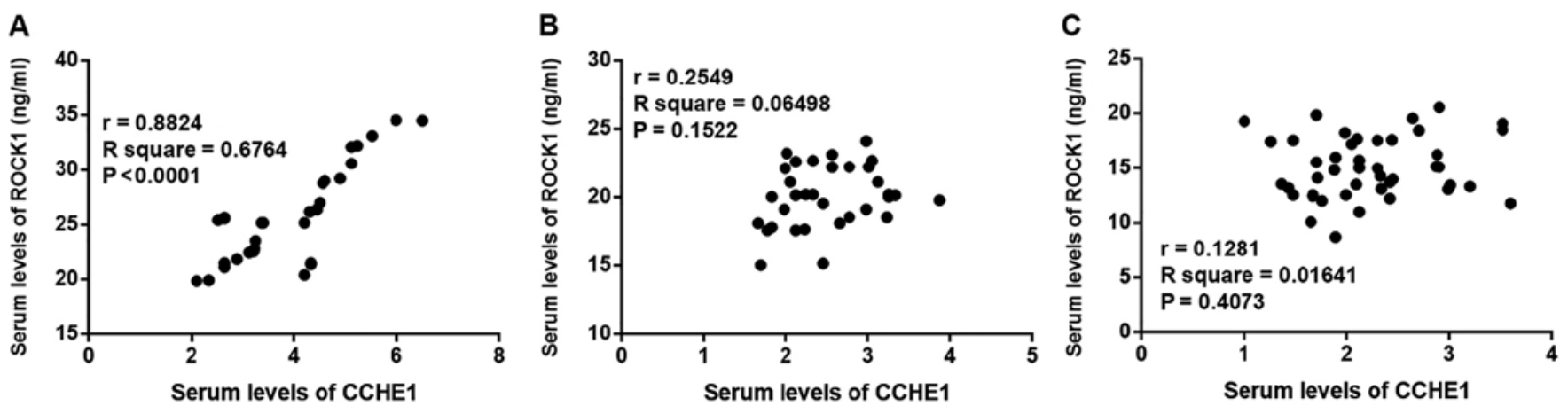

Figure 3. Serum levels of CCHE1 and ROCK1 in serum are positively correlated in patients with metastatic pancreatic adenocarcinoma. Pearson correlation coefficient analysis revealed a significant positive correlation between serum levels of CCHE1 and ROCK1 in (A) patients with metastatic pancreatic adenocarcinoma, but not in (B) patients with non-metastatic pancreatic adenocarcinoma and (C) healthy controls. CCHE1, cervical carcinoma high-expressed long non-coding RNA 1; ROCK1, rho associated coiled-coil containing protein kinase 1.

non-metastatic pancreatic adenocarcinoma and healthy controls. Selecting patients with non-metastatic pancreatic adenocarcinoma and healthy controls as true negative cases and patients with metastatic pancreatic adenocarcinoma as true positive cases, the diagnostic value of lncRNA-CCHE1 for metastatic pancreatic adenocarcinoma was evaluated by ROC curve analysis (Fig. 2). With healthy controls as true negative cases, the area under the curve (AUC) was 0.8895 (standard error, 0.03976; 95\% confidence interval, 0.8116-0.9675; $\mathrm{P}<0.0001)$. With patients with non-metastatic pancreatic adenocarcinoma as true negative cases, AUC was 0.9282 (standard error, $0.02801 ; 95 \%$ confidence interval, 0.8733-0.9831; $\mathrm{P}<0.0001)$.

Serum levels of CCHE1 and ROCK1 are positively correlated in patients with metastatic pancreatic adenocarcinoma. Pearson correlation analysis revealed a significantly positive correlation between serum levels of CCHE1 and ROCK1 in patients with metastatic pancreatic adenocarcinoma (Fig. 3A). However, the correlation between serum levels of CCHE1 and ROCK1 was not identified to be significant in patients with non-metastatic pancreatic adenocarcinoma (Fig. 3B) or in healthy controls (Fig. 3C).
CCHE1 overexpression does not significantly affect the proliferation of pancreatic adenocarcinoma cells. In the present study, Capan-2 and HPAF-II cell lines were used to perform CCHE1 and ROCK1 overexpression experiments. To investigate the role of IncRNA-CCHE1 in the proliferation of pancreatic adenocarcinoma cells, proliferation of Capan-2 and HPAF-II cells was detected by CCK- 8 assay following transfection with lncRNA-CCHE1 expression vectors. As shown in Fig. 4, compared with the control and negative control groups, lncRNA-CCHE1 overexpression did not significantly affect the proliferation of Capan-2 and HPAF-II cells.

CCHE1 overexpression significantly promotes the invasion and migration of pancreatic adenocarcinoma cells via upregulation of ROCK1. To investigate the involvement of lncRNA-CCHE1 and ROCK1 in the migration and invasion of pancreatic adenocarcinoma cells, Transwell migration and invasion assays were conducted on Capan-2 and HPAF-II cells following transfection with lncRNA-CCHE1 expression vectors. Cell migration and invasion were presented as a percentage. Compared with the control and negative control groups, lncRNA-CCHE1 and ROCK1 overexpression 

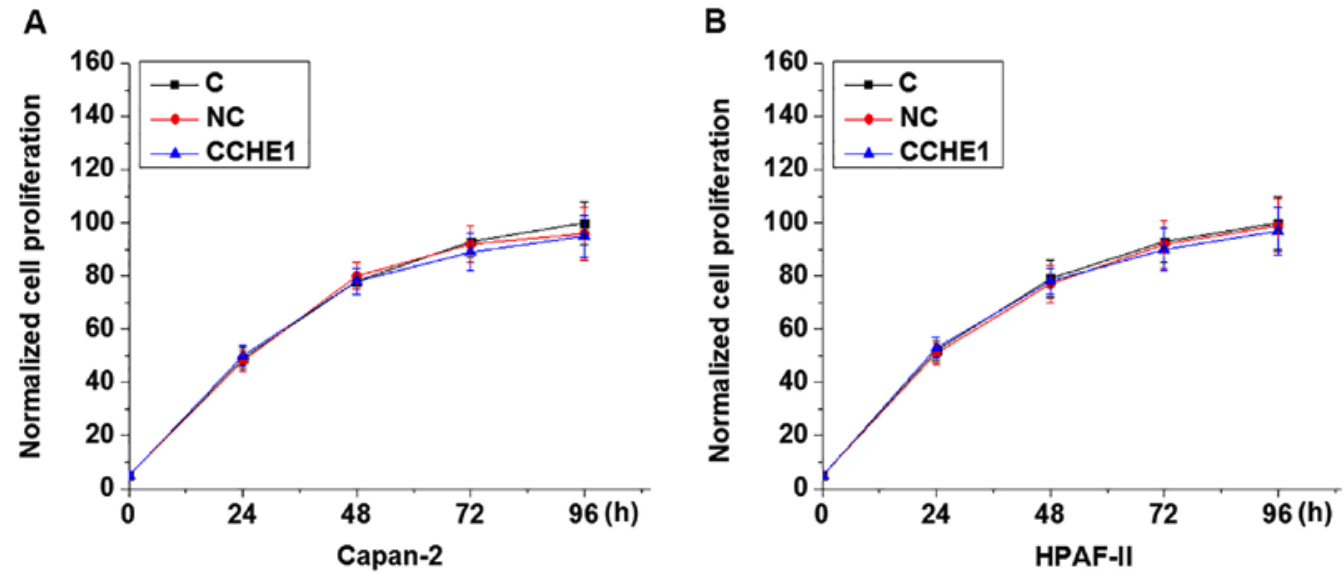

Figure 4. CCHE1 overexpression does not affect the proliferation of (A) Capan-2 and (B) HPAF-II pancreatic adenocarcinoma cells. C, control; CCHE1, cervical carcinoma high-expressed long non-coding RNA 1; NC, negative control.
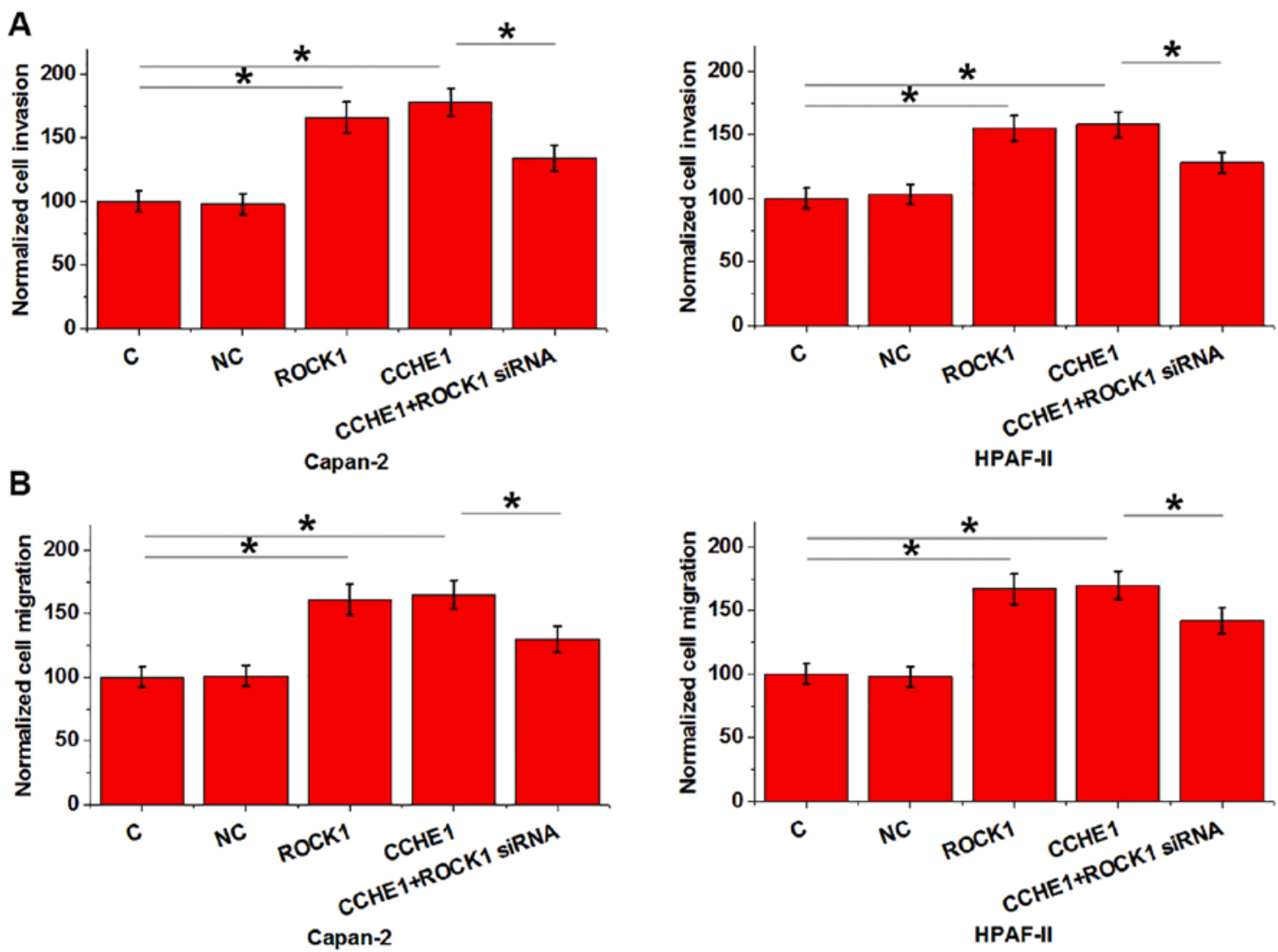

Figure 5. CCHE1 overexpression significantly promotes the migration and invasion of pancreatic adenocarcinoma cells, potentially via the upregulation of ROCK1. IncRNA-CCHE1 and ROCK1 overexpression significantly promoted the (A) invasion and (B) migration of Capan-2 and HPAF-II cells. ROCK1 siRNA-induced silencing partially reversed the enhancing effects of lncRNA-CCHE1 overexpression on cancer cell invasion and migration. "P<0.05. C, control; CCHE1, cervical carcinoma high-expressed lncRNA 1; IncRNA, long non-coding RNA; NC, negative control; ROCK1, rho associated coiled-coil containing protein kinase 1; siRNA, small interfering RNA.

significantly promoted the invasion (Fig. 5A) and migration (Fig. 5B) of Capan-2 and HPAF-II cells $(\mathrm{P}<0.05)$. In addition, ROCK1 siRNA-induced silencing partially reversed the enhancing effects of lncRNA-CCHE1 overexpression on cancer cell invasion (Fig. 5A) and migration (Fig. 5B; $\mathrm{P}<0.05$ ).

CCHE1 overexpression mediates the upregulation of ROCKI in pancreatic adenocarcinoma cells. To investigate the potential interaction between lncRNA-CCHE1 and ROCK1, the expression levels of IncRNA-CCHE1 and ROCK1 were detected by RT-qPCR and western blotting following transfection with lncRNA-CCHE1 and ROCK1 expression vectors. Compared with in the control and negative control groups, lncRNA-CCHE1 overexpression upregulated ROCK1 expression in Capan-2 and HPAF-II cell lines (Fig. 6A; $\mathrm{P}<0.05$ ). Conversely, in cancer cells with ROCK1 overexpression, no 

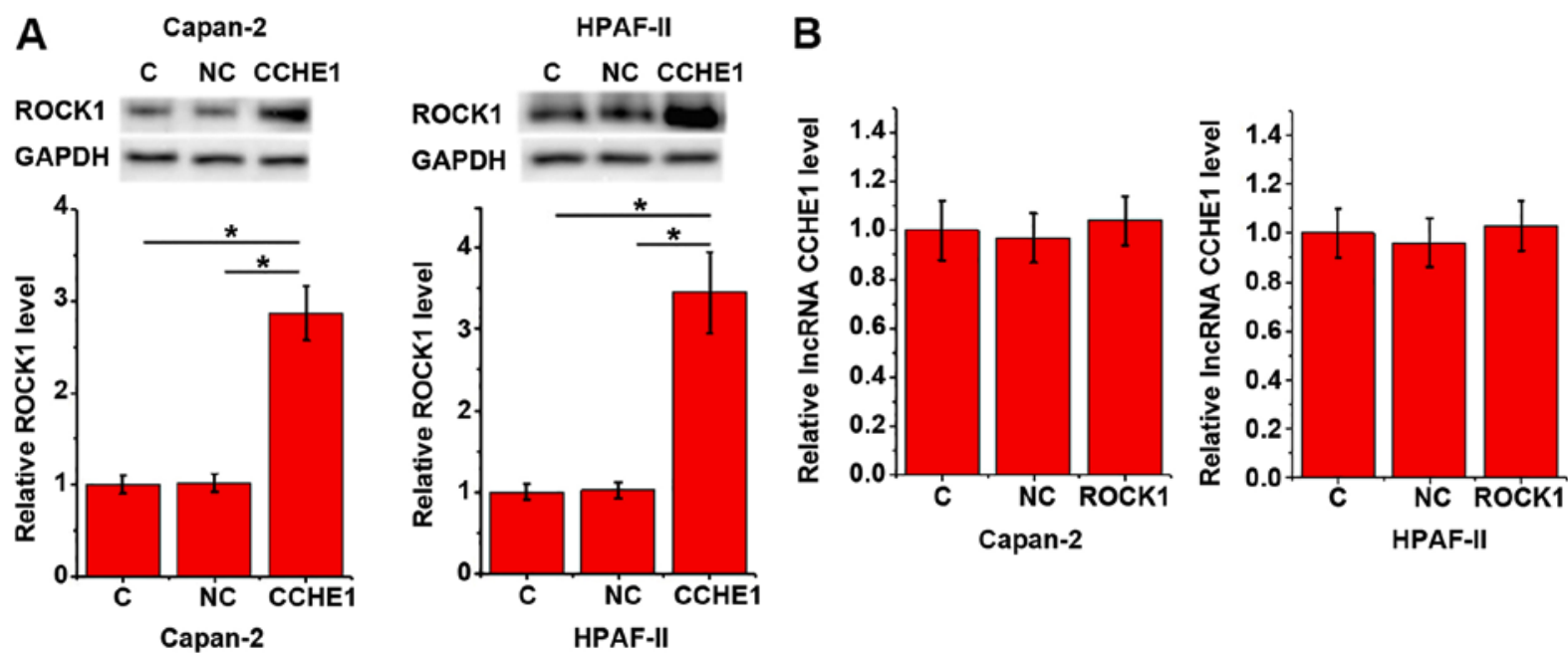

Figure 6. CCHE1 overexpression mediates the upregulation of ROCK1 in pancreatic adenocarcinoma cells. (A) Compared with the C and NC groups, lncRNA-CCHE1 overexpression resulted in upregulated ROCK1 expression in Capan-2 and HPAF-II cells. (B) By contrast, cancer cells with ROCK1 overexpression did not exhibit significantly altered expression levels of lncRNA-CCHE1. ${ }^{*} \mathrm{P}<0.05$. C, control; CCHE1, cervical carcinoma high-expressed lncRNA 1; lncRNA, long non-coding RNA; NC, negative control; ROCK1, rho associated coiled-coil containing protein kinase 1.

significantly altered expression levels of lncRNA-CCHE1 were observed (Fig. 6B; $\mathrm{P}<0.05$ ).

\section{Discussion}

Pancreatic adenocarcinoma is the most common type of pancreatic cancer. Notably, the present study demonstrated that CCHE1, which is a characterized oncogenic lncRNA in cervical and liver cancer $(12,13)$, exhibited oncogenic functions in pancreatic adenocarcinoma. The actions of lncRNA-CCHE1 in pancreatic adenocarcinoma were possibly mediated by upregulation of ROCK1.

The present study observed significantly upregulated serum levels of lncRNA-CCHE1 in patients with metastatic pancreatic adenocarcinoma but not in patients with non-metastatic pancreatic adenocarcinoma. Tumor metastasis is accompanied by a globally altered expression pattern of genes, including lncRNAs $(16,17)$. Therefore, the upregulated expression of lncRNA-CCHE1 may be mediated by the occurrence of tumor metastasis in patients with pancreatic adenocarcinoma, alternatively lncRNA-CCHE1 overexpression may induce metastasis. Combined with the observation that lncRNA-CCHE1 overexpression significantly promoted the migration and invasion of two pancreatic adenocarcinoma cell lines, this suggested that lncRNA-CCHE1 may serve an oncogenic role in pancreatic adenocarcinoma by promoting tumor metastasis. Notably, no significant alterations in the proliferation rate of the two tested cell lines were identified following lncRNA-CCHE1 overexpression. Therefore, it may be unlikely that lncRNA-CCHE1 is involved in the growth of pancreatic adenocarcinoma. However, a previous study demonstrated that lncRNA-CCHE1 expression can promote the proliferation of cervical cancer cells (12). Therefore, IncRNA-CCHE1 may serve different roles in various malignancies.

ROCK1 is involved in cancer biology mainly by regulating cancer cell motility and tumor metastasis (10). Upregulation of ROCK1 has been observed in various types of cancer, including pancreatic cancer $(18,19)$. Consistent with previous studies, the results of the present study demonstrated upregulation of serum ROCK1 in patients with pancreatic adenocarcinoma, compared with in healthy controls. ROCK1 signaling in cancer biology is transduced via interactions with different signaling molecules, inducing lncRNAs $(20,21)$. The present study revealed a significant correlation between IncRNA-CCHE1 and ROCK1 in patients with metastatic pancreatic adenocarcinoma. Additionally, lncRNA-CCHE1 was suggested to be an upstream activator of ROCK1 signaling in the regulation of migration and invasion of pancreatic adenocarcinoma cells. Notably, although serum levels of ROCK1 were upregulated in patients with non-metastatic pancreatic adenocarcinoma, no significant correlation between serum levels of lncRNA-CCHE1 and ROCK1 was observed among those patients. ROCK1 signaling may interact with multiple functional molecules in pancreatic adenocarcinoma and the crosstalk between ROCK1 and lncRNA-CCHE1 may only occur at late stages. Besides ROCK1, lncRNA-CCHE1 may also interact with other signaling molecules to regulate pancreatic adenocarcinoma cell migration and invasion, since ROCK1 siRNA knockdown only partially reversed the enhancing effects of lncRNA-CCHE1 overexpression on cancer cell migration and invasion. Notably, CCHE1 siRNA transfection was also performed, but the knockdown rate was $<60 \%$. No significant alterations in cell migration and invasion were identified following transfection (data not shown). This may be due to the low knockdown efficacy of CCHE1, or that CCHE1 knockdown has no marked effect on cancer cell migration and invasion. Future studies will try to improve the knockdown efficacy by evaluating the effects of additional siRNAs.

In conclusion, CCHE1 is likely to be an oncogenic lncRNA associated with metastasis but not growth of pancreatic adenocarcinoma. The actions of CCHE1 in pancreatic adenocarcinoma were possibly achieved via upregulation of ROCK1. The results of the present study suggested that CCHE1 may be used as a diagnostic biomarker for pancreatic adenocarcinoma, and also as a potential target to prevent pancreatic adenocarcinoma metastasis. 


\section{Acknowledgements}

Not applicable.

\section{Funding}

This study was supported by Medical and Health Technology Project of Zhejiang Province (grant no. 2016KYB323).

\section{Availability of data and materials}

The datasets used and/or analyzed during the current study are available from the corresponding author on reasonable request.

\section{Authors' contributions}

XJ and WL designed the experiments. XJ, LY and ML performed the experiments. BG, JW and $\mathrm{YH}$ analyzed data. WL drafted the manuscript. All authors approved the final manuscript.

\section{Ethics approval and consent to participate}

The present study was approved by the Ethics Committee of Taizhou Hospital of Zhejiang Province. All participants fully understood the experimental protocol and signed informed consent forms.

\section{Patient consent for publication}

Not applicable.

\section{Competing interests}

The authors declare that they have no competing interests.

\section{References}

1. Li D, Xie K, Wolff R and Abbruzzese JL: Pancreatic cancer. Lancet 363: 1049-1057, 2004.

2. Malvezzi M, Bertuccio P, Levi F, La Vecchia C and Negri E: European cancer mortality predictions for the year 2014. Ann Oncol 25: 1650-1656, 2014

3. Siegel RL, Miller KD and Jemal A: Cancer statistics, 2015. CA Cancer J Clin 65: 5-29, 2015.

4. Sohal DP, Mangu PB, Khorana AA, Shah MA, Philip PA, O'Reilly EM, Uronis HE, Ramanathan RK, Crane CH, Engebretson A, et al: Metastatic pancreatic cancer: American Society of clinical oncology clinical practice guideline. J Clin Oncol 34: 2784-2796, 2016.

5. Rahib L, Smith BD, Aizenberg R, Rosenzweig AB, Fleshman JM and Matrisian LM: Projecting cancer incidence and deaths to 2030: The unexpected burden of thyroid, liver, and pancreas cancers in the United States. Cancer Res 74: 2913-2921, 2014.

6. Hurwitz HI, Uppal N, Wagner SA, Bendell JC, Beck JT, Wade SM III, Nemunaitis JJ, Stella PJ, Pipas JM, Wainberg ZA, et al: Randomized, double-blind, phase II study of ruxolitinib or placebo in combination with capecitabine in patients with metastatic pancreatic cancer for whom therapy with gemcitabine has failed. J Clin Oncol 33: 4039-4047, 2015.
7. Amano M, Fukata Y and Kaibuchi K: Regulation and functions of Rho-associated kinase. Exp Cell Res 261: 44-51, 2000.

8. Lochhead PA, Wickman G, Mezna M, Mezna M and Olson MF: Activating ROCK1 somatic mutations in human cancer. Oncogene 29: 2591-2598, 2010.

9. Gilkes DM, Xiang L, Lee SJ, Chaturvedi P, Hubbi ME, Wirtz D and Semenza GL: Hypoxia-inducible factors mediate coordinated RhoA-ROCK1 expression and signaling in breast cancer cells. Proc Natl Acad Sci USA 111: E384-E393, 2014.

10. Rath N and Olson MF: Rho-associated kinases in tumorigenesis: Re-considering ROCK inhibition for cancer therapy. EMBO Rep 13: 900-908, 2012.

11. Li J, Song Y, Wang Y, Luo J and Yu W: MicroRNA-148a suppresses epithelial-to-mesenchymal transition by targeting ROCK1 in non-small cell lung cancer cells. Mol Cell Biochem 380: 277-282, 2013.

12. Yang M, Zhai X, Xia B, Wang Y and Lou G: Long noncoding RNA CCHE1 promotes cervical cancer cell proliferation via upregulating PCNA. Tumuor Biol 36: 7615-7622, 2015.

13. Peng $\mathrm{W}$ and Fan H: Long noncoding RNA CCHE1 indicates a poor prognosis of hepatocellular carcinoma and promotes carcinogenesis via activation of the ERK/MAPK pathway. Biomed Pharmacother 83: 450-455, 2016.

14. Kamarajah SK, Burns WR, Frankel TL, Cho CS and Nathan H: Validation of the American Joint Commission on Cancer (AJCC) staging system for patients with pancreatic adenocarcinoma: A surveillance, epidemiology and end results (SEER) analysis. Ann Surg Oncol 24: 2023-2030, 2017.

15. Livak KJ and Schmittgen TD: Analysis of relative gene expression data using real-time quantitative PCR and the 2(-Delta Delta C(T)) method. Methods 25: 402-408, 2001.

16. Smith JJ, Deane NG, Wu F, Merchant NB, Zhang B, Jiang A, Lu P, Johnson JC, Schmidt C, Bailey CE, et al: Experimentally derived metastasis gene expression profile predicts recurrence and death in patients with colon cancer. Gastroenterology 138 : 958-968, 2010

17. Chisholm KM, Wan Y, Li R, Montgomery KD, Chang HY and West RB: Detection of long non-coding RNA in archival tissue: Correlation with polycomb protein expression in primary and metastatic breast carcinoma. PLoS One 7: E47998, 2012.

18. Vigil D, Kim TY, Plachco A, Garton AJ, Castaldo L, Pachter JA, Dong H, Chen X, Tokar B, Campbell SL and Der CJ: ROCK1 and ROCK 2 are required for non-small cell lung cancer anchorage-independent growth and invasion. Cancer Res 72: 5338-5347, 2012 .

19. Shields MA, Krantz SB, Bentrem DJ, Dangi-Garimella S and Munshi HG: Interplay between $\beta 1$-integrin and Rho signaling regulates differential scattering and motility of pancreatic cancer cells by snail and Slug proteins. J Biol Chem 287: 6218-6229, 2012.

20. Wang Y, Zeng X, Wang N, Zhao W, Zhang X, Teng S, Zhang Y and Lu Z: Long noncoding RNA DANCR, working as a competitive endogenous RNA, promotes ROCK1-mediated proliferation and metastasis via decoying of miR-335-5p and miR-1972 in osteosarcoma. Mol Cancer 17: 89, 2018.

21. Wang $H$, Zhang $M$ and Sun G: Long non-coding RNA NEAT1 regulates the proliferation, migration and invasion of gastric cancer cells via targeting miR-335-5p/ROCK1 axis. Pharmazie 73: 150-155, 2018.

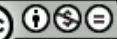

This work is licensed under a Creative Commons Attribution-NonCommercial-NoDerivatives 4.0 International (CC BY-NC-ND 4.0) License. 